\title{
Multiple Sclerosis - A Vascular Etiology?
}

\author{
Bryce Weir
}

\begin{abstract}
From the earliest pathological studies the perivenular localization of the demyelination in multiple sclerosis (MS) has been observed. It has recently been suggested that obstructions to venous flow or inadequate venous valves in the great veins in the neck, thorax and abdomen can cause damaging backflow into the cerebral and spinal cord circulations. Paolo Zamboni and colleagues have demonstrated abnormal venous circulation in some multiple sclerosis patients using non-invasive sonography and invasive venography. Furthermore, they have obtained apparent clinical improvement or stabilization by endovascular ballooning of points of obstruction in the great veins in some, at least temporarily. If non-invasive observations by others validate their initial observations of a significantly increased prevalence of venous obstructions in MS then trials of angioplasty/stenting would be justified in selected cases in view of the biological plausibility of the concept.
\end{abstract}

RÉSUMÉ: Étiologie vasculaire de la sclérose en plaques. La localisation périveineuse de la démyélinisation a été observée dès les premières études anatomopathologiques sur la sclérose en plaques (SP). L'obstruction du flux veineux ou la présence de valves veineuses inadéquates dans les grandes veines du cou, du thorax et de l'abdomen pourraient causer un reflux dommageable à la circulation cérébrale et la moelle épinière selon une hypothèse récente. Paolo Zamboni et ses collègues ont démontré la présence d'une circulation veineuse anormale chez certains patients atteints de SP au moyen l'échographie non effractive et de la veinographie effractive. De plus ils auraient obtenu une amélioration clinique ou une stabilisation de l'état des patients par dilatation endovasculaire de points d'obstruction dans les grandes veines chez certains patients, du moins temporairement. Si des observations non effractives par d'autres chercheurs valident ces observations initiales d'une prévalence significativement accrue d'obstructions veineuses dans la SP, des essais thérapeutiques d'angioplastie/endoprothèse seraient justifiés chez des patients bien choisis, à cause de la plausibilité biologique du concept.

Can. J. Neurol. Sci. 2010; 37: 745-757

There has been a recent resurgence of interest in the possibility that cerebrospinal venous outflow obstruction, venous hypertension, development of collateral channels and flow reversal might be the primary inciting factor(s) for multiple sclerosis (MS). ${ }^{1}$ The evidence should be considered bearing in mind Hill's criteria for causation as outlined by Giovannoni and Cutter: consistency and unbiasedness of findings, strength of association, temporal sequence, biological gradient, specificity, coherence of biological background and previous knowledge, biological plausibility, reasoning by analogy and experimental evidence. ${ }^{2}$ At the present state of our knowledge, despite monumental efforts, the cause is unknown.

\section{Anatomy Of Venous Outflow From The Brain And Spinal Cord}

Capillaries drain into venules $(10-20 \mu)$ that combine, almost at right angles to form larger venules within the grey matter. At a size of $50-100 \mu$ the venules lead on to the cortical surface at right angles to it. At the surface they combine in a two dimensional network. The craniospinal venous system is valveless and consists of veins and plexuses whose flow is potentially bidirectional. The superior cortical cerebral veins drain rostrally into the superior sagittal sinus. The inferior lateral cortical veins drain into the lateral dural sinus. The pattern of cortical veins is highly variable. The deep medullary and subependymal veins consist of the subependymal, internal cerebral, basal and great vein of Galen and drain the deep white and grey matter surrounding the ventricles and basal cisterns (Figure 1). The sylvian fissure contains the superficial middle cerebral veins and anterior temporal veins that drain into the sphenoparietal and/or cavernous sinuses. Interconnected with the superficial are the deep middle cerebral veins that join with the fronto-orbital, inferior striate, anterior cerebral and uncal veins to drain backwards into the basal vein of Rosenthal. This pair of veins unites with the single vein of Galen. The potential anastamoses of deep cerebral veins are numerous. Those with the superior cerebral veins can be via extracerebral or

\footnotetext{
From the Department of Surgery, University of Chicago, Illinois, USA; University of Alberta, Edmonton, Alberta, Canada.

Received April 7, 2010. Final Revisions Submitted May 20, 2010.

Correspondence to: bkaweir@shaw.ca
} 


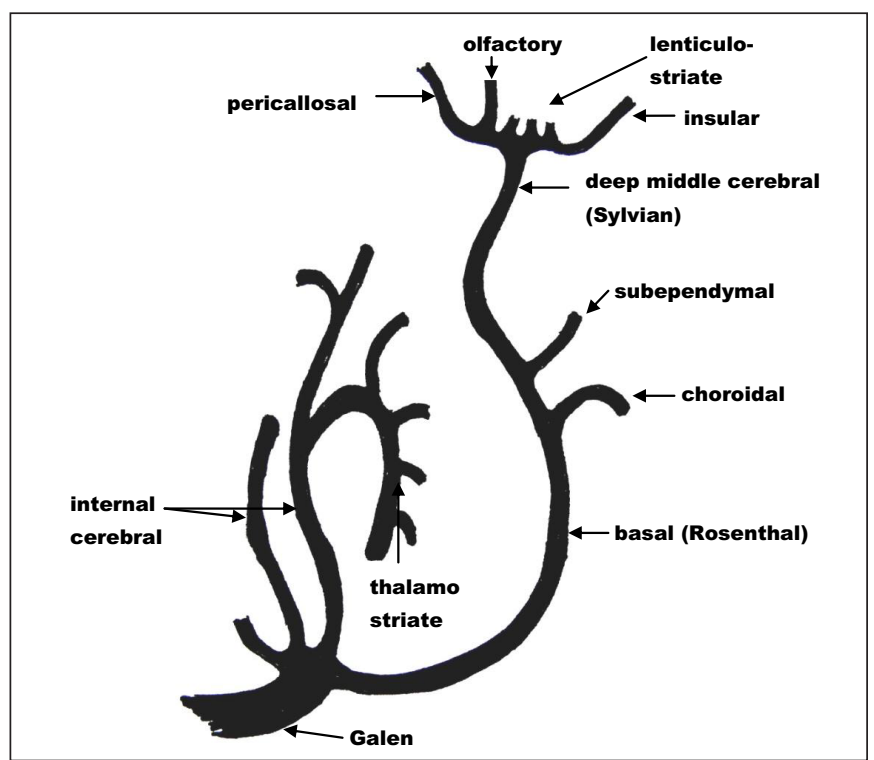

Figure 1: Deep cerebral veins on the right viewed from above and behind.

transcortical anastamoses. The deep cerebral veins can also anastamose with one another via extracerebral or intracerebral anastamoses. The deep middle cerebral veins accompany branches of the middle cerebral artery, and the basal veins accompany the posterior cerebral artery for part of their courses. The cerebral venous system has the capacity to bypass most obstructions. In the cortex the vein of Trollard on the lateral fronto-parietal cortex is joined by the sylvian veins going to the superior sagittal sinus. The vein of Labbé potentially communicates with sylvian veins and drains the lateral temperooccipital cortex into the lateral sinus. The sylvian veins interconnect the superficial and deep middle cerebral veins.

The central retinal veins drain the capillaries of the retina to enter the optic nerves on exiting the globe. The vortex veins of the choroid penetrate the sclera and merge into the ophthalmic veins, the superior ophthalmic drains anteriorly into the ethmoidal and nasofrontal veins or posteriorly into the cavernous sinus and the inferior ophthalmic vein that has a similar termination. The veins within the optic nerves may communicate with the superior chiasmatic network that drains into the anterior cerebral veins or the inferior network that drains into the basal veins.

The vein of Galen joins with the inferior sagittal sinus, both then connect to the straight dural sinus which drains posteriorly into the torcula (Figure 2). Flow in that structure divides into the two lateral sinuses each of which becomes a sigmoid sinus. The straight sinus is a common junction of both the superficial and deep venous systems. In more than $50 \%$ of cases the lateral sinuses drain the torcula asymmetrically. In draining the posterior fossa the sigmoid sinus has input from the superior and inferior petrosal sinus and veins coming off the lateral pons and medulla. Other posterior fossa veins drain mainly superiorly into the vein of Galen, the petrosal sinuses or a tentorial group of sinuses that drain superiorly into the torcula. The posterior fossa veins are classified into superficial, deep and brain stem veins (superficial petrosal vein) that drain into the superior petrosal sinus. The basioccipital and inferior petrosal sinuses drain inferiorly towards the anterior condylar confluence and internal jugular vein (IJV). A posterior communicating venous drainage exists in the occipital sinus that may connect with the sigmoid sinus or exit via the mastoid and condylar emissary veins. There is a perioccipital venous intradural ring around the foramen magnum. The basal (retroclival) sinus anastamoses with the cavernous sinus superiorly and numerous extradural venous plexuses inferiorly.

In the head the veins of the brain, the meninges (dural sinuses), the skull (diploic veins) and the various extracranial venous sinuses are joined by rich anastamotic networks. The cavernous sinus interconnects with the sphenoparietal, ophthalmic, petrosal and basioccipital sinuses.

Venous connections between dural venous sinuses of the posterior cranial fossa and the craniocervical vertebral venous system include the lateral, posterior and anterior condylar veins, the mastoid and occipital emissary veins. The anterior condylar confluence is located on the external orifice of the canal of the hypoglossal nerve (Figure 3). It is the most important connection between the intracranial cerebral venous system and the vertebral venous systems. It offers multiple connections with the dural venous sinuses of the posterior cranial fossa, IJV and the vertebral venous systems. The anterior condylar vein primarily drains into the internal vertebrovenous plexus. The posterior and

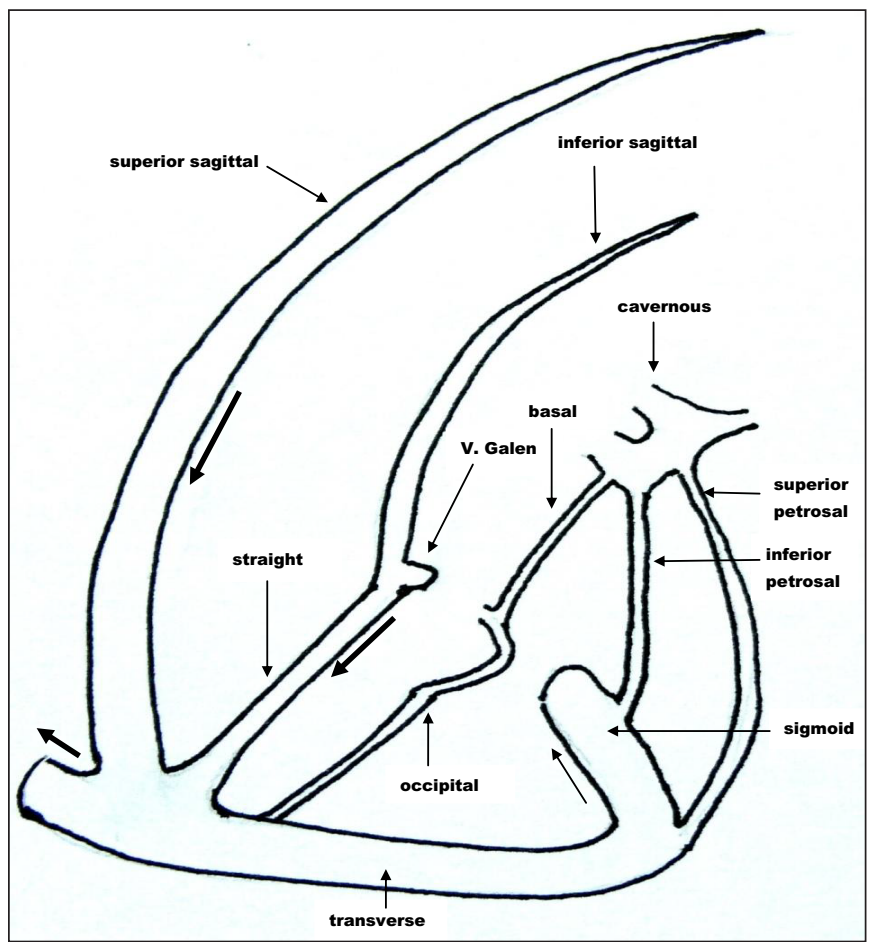

Figure 2: Venous sinuses of the brain viewed from behind and the right. 
lateral condylar veins drain into the external vertebrovenous system. There is an extradural axis that contains a continuum of venous plexuses extending from the ophthalmic to the coccygeal. In the craniocervical region it contains the basilar plexus, inferior petrosal sinuses and anterior condylar veins. There are connections between intracranial and extracranial veins at the cranial base (Batson's plexus). The suboccipital venous plexus is the cranial origin of the posterior external vertebral plexus between the dorsal muscles, it is connected to the sigmoid sinus via mastoid and condylar emissaries. The posterior internal vertebral plexus receives blood from the occipital sinus that is one of the drainage routes of the torcula.

The major routes for blood leaving the brain are from (1) sigmoid sinuses and inferior petrosal sinuses to the IJV and (2) from the venous plexus of the carotid artery, the superior jugular bulb and the IJV to the anterior condylar confluence thence to (i) veins around the vertebral arteries (ii) the venous plexus within the spinal canal (anterior internal spinal venous plexus) and (iii) the deep cervical veins. ${ }^{3}$

The head and neck veins are variable in pattern and number.

The IJVs drain blood from the brain, head and neck. The IJV begins in the posterior compartment of the jugular foramen in the skull base. Its origin is the dilated jugular bulb. It receives multiple tributaries such as the occipital, pharyngeal, facial, lingual and thyroid veins. The left vein is usually the smaller one. The IJV joins with the subclavian vein to form the brachiocephalic (innominate) vein (Figure 4).

The cervical vertebral vein partly surrounds the extracranial parts of the vertebral artery. Each begins between skull and cervical body 1 by the union of branches from the internal vertebral venous plexuses as they exit the vertebral canal. The vertebral vein crosses the posterior arch of the atlas to the foramen in the transverse process of the axis. It receives blood from condylar veins, emissary veins and multiple tributaries via the intervertebral foramina. Occasionally the venous plexus around the vertebral artery forms two trunks, anterior and posterior, instead of one. The cervical vertebral vein emerges from the transverse process foramen of the sixth cervical vertebra, is joined by the deep cervical vein and descends anterolaterally to enter the upper portion of the brachiocephalic vein medial to the entry point of the IJV that in turn is medial to the entry point of the external jugular vein. The anterior vertebral vein is formed by branches of venous plexuses in front of the vertebral bodies and the roots of their transverse processes. It lies along the ascending cervical artery and ends in the cervical vertebral vein immediately below the foramen of the sixth cervical transverse process. The deep cervical vein is found in the suboccipital triangle, it descends behind the cervical transverse processes in the company of the deep cervical artery and turns forward at the root of the neck between transverse processes of the sixth and seventh vertebrae and opens into the cervical vertebral vein. It receives blood from soft tissue of the posterior neck. The cervical end of the anterior external spinal venous plexus consists of two longitudinal and sometimes asymmetrical veins situated on either side of the midline behind the semispinalis muscles.

If it has one, the brachiocephalic vein has a single bicuspid valve. The right brachiocephalic vein receives the right IJV, internal mammalary vein, the right posterior intercostal vein, sometimes the right superior thyroid vein or a common trunk of both thyroid veins. The right lymphatic trunk also opens into it. The left brachiocephalic vein receives the thoracic duct, left IJV, the internal mammalary and superior intercostals veins on the left side. The left brachiocephalic vein is longer than the right. The two brachiocephalic veins fuse forming the superior vena cava.

The venous plexuses in and around the cervical and upper thoracic vertebrae are the: longitudinal prevertebral veins, epidural venous plexus, deep cervical and posterior intercostal veins of the posterior external plexus and basivertebral veins. The longitudinal prevertebral veins drain into the azygous venous (AZY) system via the superior intercostal vein in the

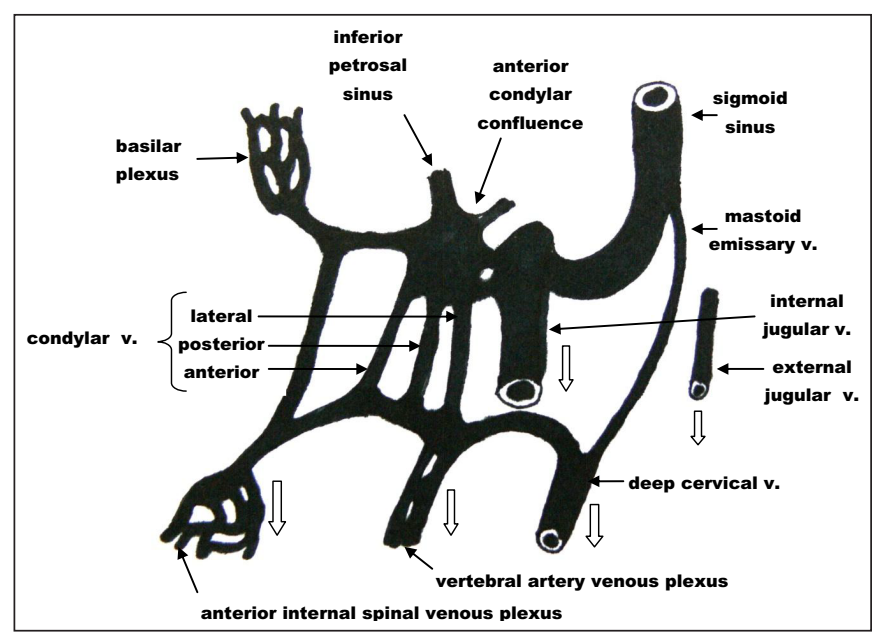

Figure 3: Right anterior condylar confluence and sigmoid sinus viewed from behind $(v .=$ vein $)$.

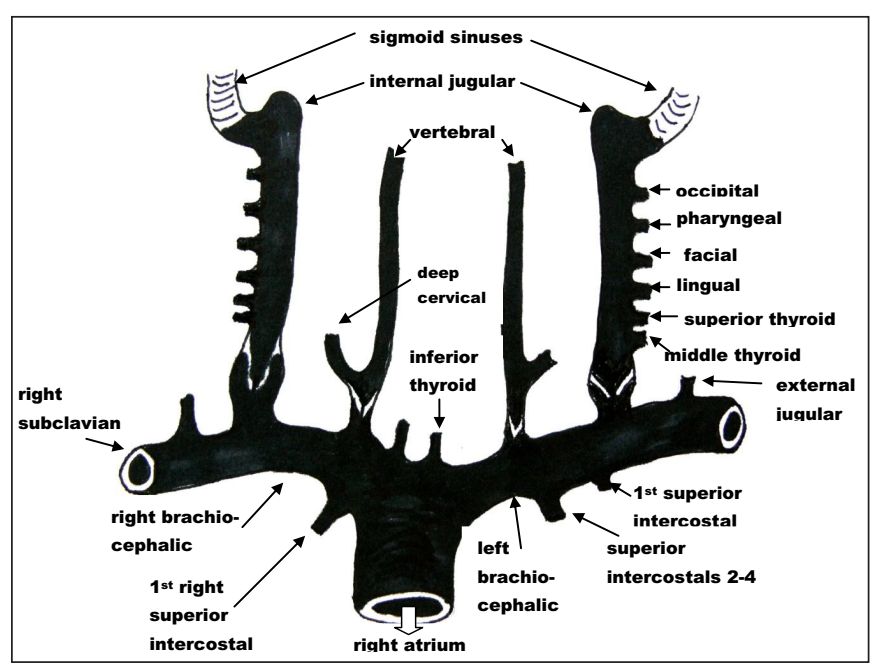

Figure 4: Cervical and thoracic viens. 
upper thorax. The deep cervical veins also anastamose with the posterior intercostal veins. The basivertebral veins are tortuous channels within the vertebral bodies that connect anteriorly with the longitudinal prevertebral veins and posteriorly with the anterior epidural venous plexus. The perivertebral venous system in the thoracic inlet is a potential collateral pathway in the case of stenosis or occlusion of the brachiocephalic veins.

The intracranial venous system communicates freely with the vertebral venous plexuses and veins. The vertebral venous plexuses anastamose with the sacral, pelvic and prostatic venous plexuses. In the skull the dura is tightly applied to the inner cranium but in the spine there is a considerable space between the dura and the fibro-osteal canal wall, which is filled with fat and the epidural venous plexus, whose capacity is estimated to be between 200 and $1,000 \mathrm{ml}$. Few or no intraspinal posterior longitudinal plexuses are found at the cervical level but became evident in the thoracic level and more voluminous and sinusoidal in the lumbar spine.

The venous plexuses extend the length of the spinal canal. They have three intercommunicating divisions; the internal plexuses lying anteriorly and posteriorly, intraspinally but extradurally, the external plexuses lying anteriorly and posteriorly to the spinal column and the basivertebral veins running horizontally within the vertebrae. The internal and external systems freely communicate with one another. The internal anterior longitudinal veins anastamose with the bilateral vertebral veins via intervertebral veins. The posterior internal vertebrovenous plexus shows great segmental and individual variability. Despite the absence of valves the preferential direction of flow during experimental infusions suggest the presence of functional 'valves' located in the thoracic posterior internal venous plexus. Anterograde flushing via the femoral veins of the vertebral venous system proceeded much faster than retrograde flushing via the superior vena cava. ${ }^{4}$

There are two intrinsic venous systems in the spinal cord. An anteromedian group drains the anterior columns and medial parts of the anterior horns of the grey matter into the anteromedial vein in front of the anterior sulcus. The remainder of the cord drains through radial veins to the longitudinally orientated coronal plexus. Seven or so medullary veins along the spinal cord drain the surface cord veins, join with epidural draining veins, and merge into intervertebral veins then posterior intercostal veins that connect with the AZY venous system.

The IJV valve is just proximal to the IJV junction with the brachiocephalic vein, situated about $2.5 \mathrm{~cm}$ from the termination, on closure it prevents reflux back into the IJV. This valve has two or three semilunar cusps. Sometimes the bulb at the termination of the IJV is bounded by valves both above and below. Distal valves in the IJVs were found in $93 \%$ of postmortem studies and in $87 \%$ of ultrasound images. 5,6 The tributaries of the IJV in the neck have valves. The external jugular vein has two valves, the lower at its entrance into the subclavian vein and the other one several $\mathrm{cm}$ proximal. They are commonly ineffective in preventing reflux. The spinal venous system is valveless. The superior vena cava, the right brachiocephalic and major AZY veins lack effective valves. The left brachiocephalic vein has a valve in $4-8 \%$ of cases. $^{7}$

The major AZY vein has only a few imperfect valves. The only veins whose flow is favoured by gravity from the spine and

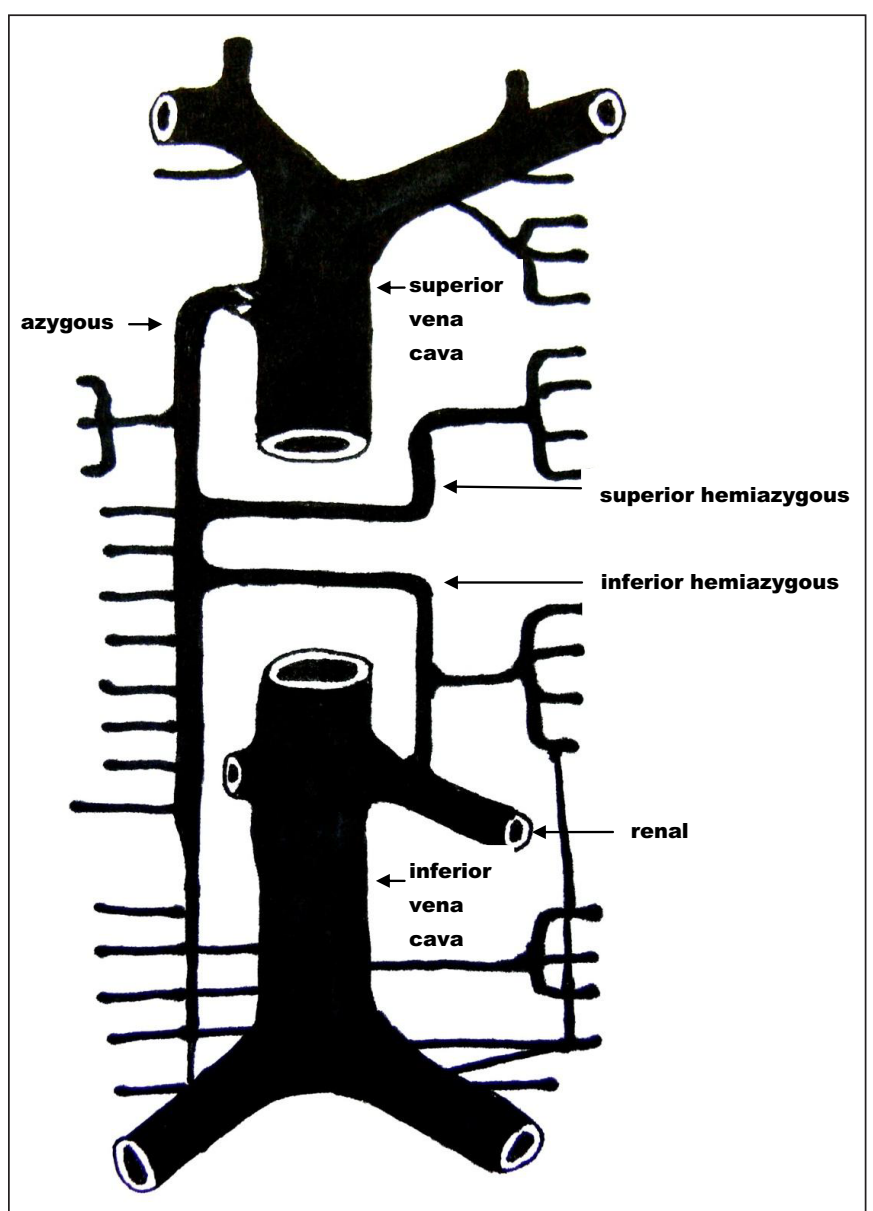

Figure 5: Azygous venous system. Intercostal and lumbar tributaries drain intervertebral veins draining spinal cord.

spinal cord are the vertebral veins, the right highest intercostals and the superior hemiAZY, all have complete valves. A cadaver study using injection and serial sectioning of the vertebral venous system confirmed that there were no bicuspid valves in intradural radicular veins. Four structures were observed that might play a similar anti-reflux role: intravenous dural folds, meandering configuration, narrowing of the radicular veins at the point of penetration of the dura mater and the variable presence of smooth muscle cells in the walls of the intradural and extradural parts of the radicular vein. ${ }^{8}$ A valve's concave surface is on the side opposite the direction of flow, the valves close to prevent reflux and open during normal venous flow towards the heart. Scapinelli, 2000, noted "Many authors have drawn attention to the possible role of increased venous pressure and slowing of the intramedullary and radicular circulation in the genesis of neurologic syndromes of various types." 9

The superior hemiAZY vein begins at the left posterior intercostals veins from the 4 th or 5 th to 7 or 8 th intercostal spaces. It crosses the midline to enter the AZY vein. The inferior hemi AZY can originate from the left renal vein, it also crosses the midline to join the AZY vein on the right. It drains the lower posterior intercostal veins. They receive posterior tributaries 
passing forward between the transverse processes of the spine. These tributaries are formed from plexuses inside the vertebral canal, plexuses anterior and posterior to the vertebral bodies and veins from muscles. In the thoracolumbar area outflow from the vertebrovenous plexuses is via the lumbar- AZY system. The AZY venous system can function as a collateral path between the superior and inferior vena cava (Figure 5).

The vertebrovenous plexuses are a freely communicating valveless system throughout the entire vertebral column, compartmentalized into intradural, epidural and extraspinal paravertebral systems. The direction of flow may change with increased intra-abdominal and intrathoracic pressure when blood would tend to be shunted back into vertebral, paravertebral and epidural venous networks. The radicular veins do not have bicuspid valves.

When there are significant obstacles to venous return from the brain or spine, collateral pathways develop to normalize intracranial venous pressures. The collateral pathways redirect blood to available venous segments outside the central nervous system. ${ }^{10}$

\section{Physiology Of Venous Outflow From Brain And Spinal Cord}

Given that the rigid skull limits the capacity of the cerebral veins to store blood it follows that the cerebral arterial inflow and venous outflow must approximate one another except perhaps in the extreme short-term. In the supine adult the flow is about 750 $\mathrm{ml} / \mathrm{min}$. In one study of healthy, supine human volunteers the venous outflow was $766 \mp 226 \mathrm{ml} / \mathrm{min} .{ }^{11}$ The venous system is estimated to contain approximately $70 \%$ of the cerebral blood volume, with about three-quarters being found within small veins and venules. ${ }^{12}$ The regional venous blood volume increases with regional blood flow. The largest contribution to regional cerebral oxygen saturation is considered to be the venous contribution. ${ }^{13}$

Pressure has been estimated at $20 \mp 5 \mathrm{~mm} \mathrm{Hg}$ in the subarachnoid veins. Forward venous flow is maintained by the residual capillary pressure from behind, the transmission pressure of the intracranial pressure (ICP) and the lower pressures in the larger thoracic veins and right atrium. Cortical venous pressure is $\mp 2-5 \mathrm{~mm} \mathrm{Hg}$ higher than the intracranial pressure and varies with it. Cerebral sinus pressure has been measured at $+1 \mathrm{~mm} \mathrm{Hg}$ in the supine position and $-3 \mathrm{~mm} \mathrm{Hg}$ in the upright. ${ }^{14}$

There is a significant linear correlation of superior sagittal sinus peak velocity and both cortical and hemispheric blood flow. ${ }^{15}$ Mean transcranial Doppler velocity in the middle cerebral artery is 50 to $60 \mathrm{~cm} / \mathrm{sec}$. A study of basal and middle cerebral veins calculated mean flow velocities of $4-17 \mathrm{~cm} / \mathrm{sec}$, much less than that of arteries. ${ }^{16}$

Venous flow reflects the pulsatility of the arterial pressure variations. The mainly jugular cervical flow showed significantly greater pulsatility than flow in the superior sagittal sinus.

The IJV are the principal venous outflow pathway in supine humans. The positioning of the IJV above the heart causes them to collapse in the upright position as blood is redirected into the vertebral veins and AZY. The perceived importance of the IJV as a venous drainage pathway has diminished lately with the realization that except in the supine position the major cerebral venous outflow is via the vertebral venous plexuses. The spinal venous system represents the major venous drainage system in humans regardless of position. Since the brain is above the heart in the sitting position a variable increase in outflow resistance from IJV collapse must occur. The vertebral venous flow is less affected in head elevation than IJV flow because of its inferior location, large capacitance and indirect connections with the superior vena cava. The cross sectional area of the IJV decreases by a factor of 6 in the standing position. A Valsalva maneuver (40 $\mathrm{mm} \mathrm{Hg}$ increase in abdominal pressure for 15 seconds) completely restored the IJV cross sectional area. ${ }^{17}$ Flow within the IJV drops about tenfold when the average subject goes from the supine to sitting position (700 to $70 \mathrm{ml} / \mathrm{min}$ ) and the crosssectional area drops from 106 to $17 \mathrm{~mm}$. Going from supine to sitting the flow in the vertebral veins increases five-fold (40 to $210 \mathrm{ml} / \mathrm{min}$ ) although the cross-sectional area remains fairly constant (10.6 to $11.9 \mathrm{~mm}){ }^{18}$ In the majority of cases one IJV has a least twice the cross-sectional area of its opposite.

Venous return is influenced by the negative intrathoracic pressure of $-5 \mathrm{~cm} \mathrm{H}_{2} \mathrm{O}$ at expiration and the even greater negative pressure of $-8 \mathrm{~cm} \mathrm{H}_{2} \mathrm{O}$ during inspiration. Venous blood is propelled from the brain by the arterial pressure from behind and sucked by increased negative pressure from the thorax during inspiration, drawing it toward the right atrium.

In a phase-contrast magnetic resonance imaging (MRI) study venous flows of individuals were heterogeneous and showed variable side dominance in paired veins and sinuses. Studies were done in the supine position despite which in some the accessory epidural drainage preponderated over normally dominant jugular outflow. ${ }^{19}$ Dural sinus blood flow studied by phase contrast MRI showed that deep inspiratory or expiratory breath holding decreased blood flow about $30 \%$ in the superior sagittal sinus and $20 \%$ in the transverse sinuses. ${ }^{20}$

Some cerebral veins are known to be collapsible, crosssectional configuration may alter depending on the transmural pressure. Internal pressure reflects up and downstream resistance to flow, flow rate, acceleration or deceleration, negative gravitational pressure and external forces (compartmental pressures - tissue, spinal fluid). Factors of importance in relation to venous velocity include partial pressure of carbon dioxide, diastolic arterial pressure and peak ventilation pressure. The venous valves play an important role in reducing the pressure applied under dynamic load conditions but understanding is rudimentary and theoretical. ${ }^{21}$ Computer modeling of the dynamics of valve opening and the control of venous flow rates is just beginning. ${ }^{22}$ The fact that venous walls are soft and compressible, and collapsible under certain conditions of reduced flow, adds to the complexity of understanding venous flow in any but a qualitative fashion. Flow through venous valves is pulsatile and separates into a central jet and vorticeal flow into the sinus pocket behind the valve cusps. Valves close when pressure from vorticeal flow exceeds pressure from the central jet flow.

\section{Neuropathology Of Multiple Sclerosis}

In 1937, Putnam proposed that MS might be caused by venous obstruction because stasis results in swelling and disintegration of myelin upstream along the vein, which in more severe lesions is accompanied by fragmentation of axis 
cylinders. He illustrated a necrotic vein within a zone of demyelination from an MS case. ${ }^{23}$ In 1939 it was observed that retrograde pressure injections into the straight sinus resulted in perivenular extravasations of the fluid that closely simulated the distribution and the shapes of plaques in multiple sclerosis. ${ }^{24}$

In the first pathological studies of MS plaques observers were divided as to whether MS plaques were always perivenular. The meticulous studies of Fog, 1965, put the matter to rest. Performing serial sections he analyzed 43 plaques. Thirty-nine followed one or two veins during their course through the white matter. Four were multivenous. Eleven were prolongations from periventricular plaques (Dawson's Fingers). No isolated plaques, which he followed from one end to the other, were independent of one or more veins. Fusiform plaques had their maximum diameter where the vein was larger. He accepted that there is concentric activity in most plaques with the most peripheral, less gliotic, region being most recent. He suggested (as had Dawson in 1916) that some substance within the vein might diffuse outward to cause the demyelination..$^{25}$

The vascular changes drew the attention of neuropathologists as far back as the 1940's and 50's. ${ }^{26}$ An early histological study examined 100 foci of demyelination. Observed vascular changes included dilatation and congestion of veins and capillaries, hypertrophy of vessel adventitia and proliferation of vessels, hyaline degeneration of vessel walls and thrombosis within vessels. Perivascular hemorrhage was sometimes seen in the initial lesions but not subsequently. ${ }^{27}$ Adams (1987), summarized the vascular aspects of MS. Periventricular plaques originated around veins, not ependyma. Perivenous cuffing with lymphocytes (both $\mathrm{T}$ and $\mathrm{B}$ cells) were common. $\mathrm{T}$ cells were sparse at the periphery of the demyelination. The vein walls were sometimes involved in an inflammatory process. In $70 \mathrm{MS}$ brains, 21 showed vein wall damage: intramural fibrinoid deposits 5, recent perivenous hemorrhages 10, mixed recent and old hemorrhages with siderocytes 2 , perivenular iron deposition 21 , venous thrombosis or encrustations 4 . In 20 of the periventricular iron cases it was related to the veins or at the edge of the plaques, this seemed to be a specific feature of MS. Prolonged damage to the vein led to collagenization. An excess of reticulin fibers was thought to indicate reparative venular thickening. Areas of the brain where plaques most commonly began drained mainly into the Galenic or petrosal systems. The centrifugal spread of plaques from the periventricular or peraqueductal regions supported the hypothesis that MS is an outwardly progressing disease beginning in the veins of the subependymal ventricular system..$^{28} \mathrm{He}$ went on to compare his MS cases to an equal number of control brains. Hemosiderin deposits in the brains of controls were occasionally found $(6.3 \%)$ but were distributed diffusely and only in the presence of coexisting cardio- or cerebrovascular disorders. By contrast $30 \%$ of MS cases showed this evidence of vein damage. Hemosiderin deposits were common in the substantia nigra and pigmented nuclei in all. The MS veins showed intramural fibrinoid (7\%), recent hemorrhage $(17 \%)$, thrombosis $(6 \%)$ and collagenized thickened walls (19\%) whereas none of the controls showed these changes. In active MS cases hemosiderin was sometimes found in areas of intense edema and myelin pallor, often associated with marked perivenular lymphocytic cuffing. He wrote "...venous permeability seems to be increased in and around active MS plaques, as shown by the oedema, fibrin and complement seen in such lesions, by postmortem permeability studies, by magnetic resonance imaging, by scintigraphy and, in periphlebitis retinae, by vitreous fluorophotometry ... vascular (venous) damage is an important factor in the pathogenesis of multiple sclerosis ..." 29

A significant recent observation was that the earliest cellular manifestations of MS may be apoptosis of oligodendrocytes followed by the transformation of microglia (resident brain macrophages) into scavenging macrophages. These changes were observed in 7 of 12 patients who died shortly after the onset of a relapse..$^{30}$

The heterogeneity of multiple sclerosis lesions may imply different pathogenetic mechanisms of demyelination. Patterns of primary oligodendrocyte dystrophy suggest toxin- induced demyelination rather than autoimmunity. ${ }^{31}$ The pattern of preferential loss of myelin-associated glycoprotein and apoptotic -like oligodendrocyte destruction shows prominent nuclear expression of a hypoxia inducible factor similar to what is seen in acute white matter strokes. ${ }^{32}$ It has been suggested that some MS lesions are mediated by mitochondrial dysfunction induced by inflammatory mediators such as reactive oxygen and nitrogen species, which result in histotoxic hypoxia. ${ }^{33}$

Dye infusions provided evidence for breakdown of the bloodbrain barrier in the 1960s. The integrity of the blood-brain barrier depends on interendothelial cell tight junctions, perivascular cells, astrocytic end-feet and two distinct basement membranes. The disruption of the blood brain barrier is an early feature of white matter lesions where plasma protein leakage may be found. Fibrinogen immunoreactivity was detected in blood vessel walls of both normal appearing grey and white matter. Breakdown of the blood brain barrier was less marked in grey matter lesions. ${ }^{34,35}$ Dysregulation of the blood-brain barrier and transendothelial migration of activated leukocytes are among the earliest cerebrovascular abnormalities and parallel the release of inflammatory cytokines/chemokines. ${ }^{36}$

The immune cells secondarily recruited need to traverse the blood brain barrier composed of specialized endothelial cells interconnected by complex tight junctions. Immunocytochemical and confocal microscopic studies of tight junction proteins were performed on blood vessels. Abnormal tight junctions were found in both secondary progressive and primary progressive MS brains, being most frequent in active white matter lesions in primary progressive cases. Abnormal tight junctions were also noted in the blood vessel endothelium of normal appearing grey matter. ${ }^{37}$ After penetrating this barrier, cells may accumulate within the perivascular space that is lined by two layers of extra-cellular matrix molecules, the vascular and the parenchymal, or proceed through both layers of these basement membranes. The loss of blood brain barrier integrity may be an early crucial event in the pathogenesis of MS lesions.

Matrix metalloproteinases have the capacity to degrade extracellular matrix components thereby influencing leucocyte infiltration, axonal degeneration and astrogliosis. In normal white matter throughout the brain, matrix metalloproteinase-19 is expressed by microglia. It is intensely expressed in highly active demyelinating MS lesions. Parenchymal and peri-vascular myelin-laden macrophages are strongly immunoreactive for matrix metalloproteinase-19. ${ }^{38}$ One mechanism by which 
Interferon- $\beta$ could favourably influence the clinical course of MS may be by raising the level of tissue inhibitors of matrix metalloproteinases. ${ }^{39}$

Iron accumulates in the brains of patients with many different types of degenerative brain diseases. There are several different forms of iron in the brain. Heme iron is found in hemoglobin and some peroxidases. Heme-oxygenase- 1 is a microsomal enzyme inducible by stress that protects cells from oxidative injury. It participates in the cleavage of the heme ring producing biliverdin, $\mathrm{CO}$ and ferrous $\mathrm{Fe}$. The iron then becomes part of the intracellular pool being stored in ferritin or released by an iron exporter. Nonheme irons include low-molecular weight complexes, metalloproteins including transferrin (iron transport), ferritin (iron storage) and hemosiderin (degradation product), as well as ionic iron. Ferritin stores excess iron atoms not immediately involved with metabolic activities. The water insoluble hemosiderin stores iron in situations of iron overload such as occur following hemorrhage. Normally iron is found throughout the brain with twice to four times as much in the basal ganglia as the cortex, the same is true for ferritin. Transferrin levels are much lower than ferritin. Of all types of neural cells oligodendrocytes, throughout the brain, stain most strongly for iron, ferritin and transferrin. ${ }^{40}$ Some forms of iron can participate in the generation of free radicals.

Iron metabolism is essential for myelin formation and oxidative phosphorylation. Iron dysregulation may play a role in the pathogenesis of MS. In MS and control brains, Perl's stain was used to detect non-heme iron present as hemosiderin. Positive iron reactions were found only around MS plaques. Myelinated white matter near the lesions contained numerous iron-laden ovoid bodies and axons staining positive for iron. Positive reactions were also found in grey matter blood vessels near the lesions. It was speculated that the iron came from extravasated blood.$^{41}$ Early studies with standard Perl's stain for iron in MS yielded conflicting results. A modified diaminobenzidine enhanced Perl's stain, utilizing a variety of agents to increase the accessibility of tissue iron to the histochemical reagents, demonstrated iron stained microglia and macrophages in MS brains. This was in addition to its normal deposition in oligodendrocytes and myelin. ${ }^{42}$

Antioxidant enzymes, including superoxide dismutase 1 and 2 , catalase and heme-oxygenase 1 , are markedly upregulated in active demyelinating lesions. They are particularly expressed in hypertrophic astrocytes and myelin-laden macrophages. This suggests an adaptive defense response to reactive oxygen species. ${ }^{43}$

Many thousands of articles on experimental autoimmune encephalomyelitis (EAE) have guided the thinking about MS. The essential distinction between EAE and MS is that the cause of the autoimmunity in EAE is known (immunization with myelin antigens or insertion of transgenes to generate encephalitogenic T lymphocytes) whereas the cause of MS is not. ${ }^{44}$ The incidence of EAE was $71 \%$ in mice with normal iron levels, $62 \%$ in iron-overloaded mice and $0 \%$ in iron-deficient mice. ${ }^{45}$ The iron chelator, Deferiprone, suppressed the disease activity of EAE. Mice that showed this suppression had significantly fewer inflammatory cell infiltrates. However, the reduction in T-cell (anti CD3 staining) did not reach significance. ${ }^{46}$

\section{Imaging Studies Of Multiple Sclerosis}

By perfusion MRI cerebral blood flow was found to be decreased in normal appearing white matter of clinically isolated syndrome and relapsing-remitting MS and subcortical normal appearing grey matter in the latter form. ${ }^{47}$ Quantitative perfusion mapping using MRI techniques compared MS and control patients. Regions of lower perfusion in predominantly grey matter were observed in primary and secondary progressive cohorts, especially in the thalamus. Increased white matter perfusion was seen in relapsing remitting and secondary progressive cohorts. ${ }^{48}$ Perfusion MRI was used to study normal appearing grey and white matter in MS patients. ${ }^{49}$ Using apparent diffusion coefficient as well as conventional MRI parameters and serial studies of the same lesions, changes in perfusion parameters were detectable not only prior to the blood brain barrier breakdown but also prior to increases in the apparent diffusion coefficient. This suggested that inflammation accompanied by altered local perfusion preceded alterations in the blood brain barrier. ${ }^{50}$

Some investigators detected abnormal, multiple foci of increased signal intensity on T2 weighted images, most prominent in the periventricular white matter, in MS compared to controls. Decreased signal on T2 in the putamen and thalamus was seen in more than half of MS patients. This was possibly due to increased iron content. ${ }^{51}$ While T2 hypointensity has been described in grey matter of MS patients and linked to physical disability that this is iron awaits pathological confirmation. Advanced MRI measures of iron deposition (R2, R2*, R2' relaxometry, 3T images and other approaches are only beginning to be applied. ${ }^{52} \mathrm{~A}$ newly developed quantitative MR technique, magnetic field correlative imaging, was used to study iron in the deep grey matter of 17 relapsing-remitting MS cases and 14 agematched controls. The magnetic field correlation was significantly increased in MS by $24 \%$ in the globus pallidus, $39.5 \%$ in the putamen and $30.6 \%$ in the thalamus. The increased iron deposition in deep grey matter correlated positively with the total number of MS lesions. ${ }^{53}$ Twenty-seven clinically definite MS patients had pre- and post-contrast MRI studies, T-1, T-2, FLAIR, susceptibility weighted imaging at 1.5,3 and 4T. A total of 75 lesions were only seen on conventional imaging, 143 only on susceptibility weighted and 204 on both. A moderate linear correlation was established between signal intensity and iron content (phase). Iron was detected in MS lesions, basal ganglia and thalamus. ${ }^{54} \mathrm{~T} 2$ hypointensity was more marked in the deep grey matter of MS patients compared to controls. ${ }^{55}$ Basal ganglia R2* relaxation rates increase in parallel with age, disease duration, brain atrophy and were significantly higher in relapsing-remitting than clinically isolated syndrome. Long-term follow-up will establish if increased iron is the cause of disease progression or just an epiphenomenon. ${ }^{56}$ Magnetic resonance imaging at 7 Tesla produced high-resolution gradient-echo phase images that quantified the local field shifts from iron in the basal ganglia and lesions. Phase images showed contrast in $74 \%$ of 403 plaques, increasing the lesion count by more than $30 \%$ and demonstrated distinct peripheral rings and close association with vasculature.$^{57}$ Venous hemodynamic abnormalities were used to select a group of MS patients who underwent susceptibility- 
weighted MRI. Iron deposition showed a modest-to-strong association with lesion volume accumulation and atrophy development..$^{58}$

Magnetic resonance imaging images at $1.5 \mathrm{~T}$ with venographic studies obtained by $3 \mathrm{D}$ gradient-echo techniques identified a central vein in almost all periventricular lesions and focal deep white matter lesions. The ovoid shape and orientation along the long axis of veins correlated with the course of the veins. ${ }^{59}$ The use of 7 Tesla MRI has provided proof of the perivascular distribution of MS plaques. ${ }^{60}$ Markedly enhanced detection of microvasculature involvement associated with most of the MS plaques was noted with 7 Tesla compared to 3 Tesla.

Neck MRI venography has not been found to have significant value in the diagnosis of "chronic cerebrospinal venous insufficiency", 61

Spinal cord plaques in MS cases visualized as hyperintense regions on T2 studies, were peripherally situated, occupied less than half the cross-sectional area of the cord and were less than two vertebral segments in length. Seventy-five percent were cervical and $25 \%$ were thoracic. ${ }^{62}$

\section{Sonography In Multiple Sclerosis}

Late in the 1980s transcranial colour-coded duplex sonography was developed. It allows a combined visualization of intraluminal blood flow and parenchymal structures. ${ }^{63}$ Venous flow velocities are higher in females, decrease with age and are generally symmetrical between paired structures. Venous pulsatility (systolic/diastolic ratio) is higher in dural sinuses than in veins and increases with age. ${ }^{64}$ The venous Doppler signal is a continuous spectral waveform of low pulsatility and is modulated by respiratory and cardiac cycles. In normal subjects deep middle cerebral veins were identified in $88 \%$, the basal veins in $97 \%$, the straight sinus in $60 \%$ and the transverse sinuses in $42 \%$. The detection rate fell with increasing age. Velocities fell from sinuses to basal veins to deep middle cerebral veins. ${ }^{65}$ During deep inhalation the IJV may collapse. A systematic ultrasound analysis of venous outflow patterns found that in the supine position $72 \%$ of volunteers had a total IJV volume flow of $>2 / 3$ of total venous outflow, $22 \%$ showed flow $>1 / 3$ and $<$ $2 / 3$, and $6 \%$ had flow $<1 / 3$. In the sitting position blood is diverted from the IJV via the anterior condylar confluence into the deep cervical veins, the vertebral veins and the intra- and extra-spinal compartments. ${ }^{66}$

From the early days of sonography the significance of IJV valve competence to prevent venous reflux has been recognized. ${ }^{67,68}$ Using air contrast ultrasonography IJV valves were observed in $96.8 \%$ of normal subjects. Retrograde venous flow occurred in 38.4\%; $20 \%<50$ years and $38.8 \%>50$ years, it was more frequent in men than women therefore the age and sex distribution were the opposite of MS. ${ }^{69}$ The extent of venous reflux depends on the pressure gradient across the valve, the adequacy of collateral veins and the presence and competence of venous valves. ${ }^{70}$ Internal jugular valves are seen in $96.8 \%$ of the general population. In 93-99.1\% of cases the valves are bicuspid. The valves close once during each diastole when the right atrium sends a surge of blood back into the entering veins. Without a competent IJV valve a sustained and prolonged retrograde transmitted venous pressure could have adverse effects on cerebral function (transient global amnesia, transient monocular blindness, cough headache and primary exertional headache). Real time sonography can show the opening and closing of the two cusps of the vertebral venous valves at the junction of the vertebral and brachiocephalic veins. The valves move synchronously with the IJV valves. If there is increased intrathoracic or intra-abdominal pressure from events such as coughing or positive end-expiratory pressure ventilation, incompetent valves can fail to prevent backflow into the brain and spinal cord thereby increasing intracranial pressure. ${ }^{71}$ Internal jugular vein valve closure was assessed using B-mode in 100 IJV valves in 50 healthy volunteers, closure was monitored during a pressure controlled Valsalva maneuver by colour duplex. Retrograde flow through insufficient valves was seen in $29 \%$ of valves. Backward flow through competent valves lasted $0.46 \mathrm{~s}(0.22-0.778 \mathrm{~s})$ and peak velocity was $26.2 \mathrm{~cm} / \mathrm{s}(12-65$ $\mathrm{cm} / \mathrm{s})$. In insufficient valves duration of backflow was $2.66 \mathrm{~s}$ (1.23-6.15 s) and peak velocity was $89.5 \mathrm{~cm} / \mathrm{s}(25-160 \mathrm{~cm} / \mathrm{s})$. On $\mathrm{B}$ - mode imaging only a few cases of the 'insufficient' valves actually showed immobile, frozen valves. ${ }^{72}$

Menegatti and Zamboni use the same ultrasound machine to examine the cerebral veins and sinuses by means of transcranial colour coded sonography at $2.5 \mathrm{Mhz}$ and the IJV and vertebral veins by means of echo-colour-Doppler operating at $7.5 \mathrm{Mhz}$ or higher. They defined reflux as venous flow reversal from the physiological direction for a duration $>0.88 \mathrm{~s}$ in the extracranial pathway and $>.50 \mathrm{~s}$ in the deep cerebral veins. In about $50 \%$ of MS patients pathological venous reflux within the deep cerebral veins and dural sinuses occurred during the activity of the thoracic pump. Such refluxes were absent in the deep cerebral veins of healthy subjects and only $7 \%$ of normals showed venous reflux in the transverse sinus. ${ }^{18}$

\section{Venous Hypertension And Flow Reversals From Non- Multiple Sclerosis Conditions}

If venous obstruction, the establishment of collateral channels, reverse flow and venous hypertension caused demyelination why has this been missed in the many diseases other than MS where these venous changes occur? Why has chronic recurring demyelination not been observed in pial arteriovenous fistula, dural sinus thrombosis, deep cerebral vein thrombosis, cranial or spinal dural arteriovenous fistula, and arteriovenous malformations? Does it only occur if the obstructions are outside the cranium and the spinal canal? Does it not occur if the changes are acute or of insufficient duration? Does it only occur in certain age groups? Does it only result if additional factors are active? Or has clinical, radiological and pathological surveillance not been sufficiently astute or long lasting to detect demyelination and relapsing-remitting symptoms?

Pseudotumor cerebri is a rare complication of bilateral radical neck dissection and sacrifice of both IJV and a single case has been reported after unilateral sacrifice. One affected patient was reported in whom unilateral IJV loss caused prominently increased flow in the contralateral $\mathrm{IJV}^{73}$ Other cases of loss of both IJV have not apparently resulted in chronic demyelination..$^{74,75}$

While the underlying renal-disease might preclude long-term survival it would be intriguing to know if the substantial and increasing population of patients with end-stage disease, who 
have dialysis catheters causing IJV and subclavian vein stenosis/occlusion, develop deep cerebral venous reflux. Stenosis from simple cannulation of the IJV has been reported in $0-10 \%$, with stenosis being defined as a $50 \%$ or greater reduction in venous diameter, with or without collateral vessel enlargement. ${ }^{76,77}$ Venography was employed in 69 patients prior to percutaneous placement of a tunneled right IJV catheter. In $42 \%$ there was an unexpected stenosis and /or angulation of the vein. If catheters had been previously placed the incidence was $65 \%(15 / 23)$, although in virgin cases such abnormalities were noted in 30\% (14/46). This may reflect a high incidence of such findings in the "normal' population. ${ }^{78}$ Of 235 patients in hemodyalysis, 133 underwent venography in a 14-month-period because of access problems, $41 \%$ had evidence of significant IJV and/or subclavian vein stenosis. The rate of stenosis increased if there had been prior catheterizations and also with a longer placement time. In cases with stenoses the catheters had been in place an average of 43 months. ${ }^{79}$ A patient with a peripheral hemodialysis shunt presented with headache, memory loss and gait difficulty. Cranial computed tomogram (CT) showed enlarged posterior fossa veins. Duplex sonography showed flow reversal in the IJV. The abnormalities improved after surgical ligation of the hemodialysis shunt. ${ }^{80}$ Another patient with a malfunctioning dialysis shunt showed on sonography turbulent and reversed flow in the IJV and occlusion of the brachiocephalic vein. Angiography showed reversal of flow up one IJV, across the transverse sinuses and caudal descent down the contralateral IJV. ${ }^{81}$

Three patients with stenosis of one brachiocephalic $\mathrm{V}$ were shown to have ipsilateral sluggish flow in the IJV with compensatory retrograde flow through the ipsilateral inferior petrosal sinus to the cavernous sinus. Two of the patients had symptoms of vertebro-basilar insufficiency. ${ }^{82}$

Internal jugular vein valve incompetence during Valsalva maneuver was found in $60 \%$ of chronic obstructive pulmonary disease patients and in $100 \%$ of primary pulmonary hypertension cases as opposed to $27 \%$ of healthy controls. ${ }^{83}$ These authors previously suggested that primary exertional headache might be caused by IJV valve incompetence, it was present in $70 \%$ of such patients and only $20 \%$ of controls. ${ }^{84}$ Transient global amnesia, the syndrome of inability to retain new information or recall past events during minutes or hours, is of unknown etiology. In one series of 20 such patients and 20 matched controls venous valvular insufficiency, uni- or bilateral, during Valsalva maneuver was documented in $85 \%$ of patients and $45 \%$ of controls. The mean duration of the insufficiency jet was the same although patients with a trigger for their global amnesia had longer insufficiency $(3.84 \mathrm{sec})$ than those without triggers $(2.55$ sec) ${ }^{85}$ It remains a matter of debate as to whether the evidence regarding IJV incompetence is circumstantial or definitive in proving the etiology of this syndrome..$^{86}$

Occlusion of the jugular bulb catheter after extracorporeal membrane oxygenation resulted in reduced superior sagittal sinus blood flow velocities. Persistent reductions in sinus velocity were associated with a higher risk of cerebrovascular injury. Alterations in cerebral venous drainage are common in newborns treated with this modality which ultimately results in the loss of an IJV. ${ }^{87}$

The introduction of shunts to treat hydrocephalus more than half a century ago has reduced the mortality rate from 80 to 15 $20 \%$. The ventriculoatrial shunts usually involved a catheter running down the IJV, necessarily keeping the valves from closing completely, and lodging in the right atrium where sometimes they became the site of infections and thrombi. Some patients developed complete obstruction of brachiocephalic veins and evolved massive collateral channels to the AZY vein and superior vena cava. Such patients have a plethora of clinical courses but the literature seems silent on any pathological or radiological evidence of disseminated cerebral plaques. ${ }^{88}$

Myelopathies have been associated with venous stenoses and compressions for decades but no direct analogies to MS have been drawn. Increasing intraspinal venous pressure was thought to result in numerous paraplegias and quadriplegias due to multiple venous anomalies, most frequently stenoses of the IJV, left renal, left iliac, AZY and brachiocephalic veins. In one series of 80 patients, $60 \%$ had at least two and $38 \%$ at least three stenoses. ${ }^{89}$

\section{Retinal Venous Pathology}

There is ophthalmological evidence to support venous blockage/reflux/stasis as a potential cause of MS. Up to $50 \%$ of MS patients develop optic neuritis at some point in the course of their disease. Wilbur Rucker first described sheathing of retinal veins in 1944. Sheathing is a fine, opaque border along retinal veins. It results from thickening of venous walls and may persist for years. More than 500 cases had been reported by 1972. The nerves of the retina are not myelinated. ${ }^{90}$ Enlarged retinal veins have been reported, albeit infrequently, in MS. ${ }^{91}$ In a series of 50 patients with acute optic neuritis, vascular abnormalities were detected in 28\%; flourescein leakage in $20 \%$, perivenous sheathing in $12 \%$, cells in the vitreous in $12 \%$ and anterior chamber $8 \% .{ }^{92}$ Given the absence of myelin in the retina these finding suggest the existence of a vascular mechanism in the pathogenesis of MS.

\section{Cerebral Venous Outflow Obstruction/Reflux Causing Multiple Sclerosis Theory}

In 2006, Paolo Zamboni published his hypothesis that MS might be analogous to lower extremity lipodermatosclerosis and ulceration, the end stage of varicose vein disease. Both abnormalities might result from venous hypertension, stasis and flow reversals. The lower extremity ulcers in chronic venous disease are surrounded and sometimes preceded by brownish pigmentation, evidence of increased tissue iron originating from extravasated erythrocytes. He opined that there were other etiopathogenetic factors in common between the two diseases including the presence of adhesion molecules and leucocyte activation, macrophage migration / infiltration, perivascular iron laden macrophages, hyperactivation of matrix metalloproteinases, hypoexpression of tissue inhibitors of matrix metalloproteinases, local iron overload and fibrin-cuffs around microvessels. The essence of his theory is that venous obstruction/reflux increases trans-mural pressure, facilitates erythrocyte diapedesis and perivenous iron accumulation that in turn can generate free-radicals, cause oxidative stress and tissue breakdown. ${ }^{1}$ Risk factors for lower extremity venous disease not shared by MS are increasing age, obesity, pregnancy, prolonged 
standing and greater height. Venous valve incompetence is the key to the development of venous hypertension in the lower extremities. It has been shown experimentally that venous hypertension can destroy valves and reduce their number. It also results in valves becoming infiltrated by inflammatory cells and the expression of intercellular adhesion molecules. ${ }^{93}$

In 2007, Zamboni and his group reported on 58 relapsingremitting and 31 secondary progressive MS cases and compared them to 60 matched controls. Transcranial colour-coded Doppler sonography established the velocity and direction of blood flow in the deep middle cerebral veins and transverse sinuses. Peak systolic and diastolic velocities were used to calculate a resistance index (peak systolic - peak diastolic/peak systolic velocity). It was greatly elevated in the MS group $(1.1 \pm 0.08)$ compared to controls $(0.48 \pm .04)$ and this was interpreted to indicate a highly significant impedance to cerebral venous flow in MS. Measurements were made in the sitting and supine positions. Deep middle cerebral venous flow was monodirectional in $100 \%$ of controls but only $35 \%$ of MS cases. Venous flow in the transverse sinuses was bi-directional in $30 \%$ of MS cases and $13 \%$ of the controls. Reflux in the deep middle cerebral veins occurred in none of the controls but $38 \%$ of the MS cases. Deep breathing (activation of the thoracic pump) accelerated venous flow. Flow was considered bidirectional when it deviated from its physiological direction toward the heart for $<.5 \mathrm{sec}$ and diagnosed as reflux flow if the phase of reversed flow was $>.5 \mathrm{sec}$. In normals, venous flow is monodirectional, laminar, with peak systolic velocity of 27 $\mathrm{cm} / \mathrm{sec}$ and diastolic of $16 \mathrm{~cm} / \mathrm{sec}$. In the MS population peak diastolic velocity was negative $(-1.3 \mathrm{~cm} / \mathrm{sec})$. In a sub-group of 34 cases with reflux detected in the deep middle cerebral veins, the peak diastolic rate was increasingly negative $(-15.3 \mathrm{~cm} / \mathrm{sec})$. Given that MS plaques are venocentric and that significant hemodynamic alternations occurred in veins anatomically related to plaque location these findings of abnormalities in cerebral venous outflow are consistent with Zamboni's hypothesis. The reversal of normal flow in a vessel could promote expression of surface adhesion molecules and an inflammatory reaction. Macrophage and T-cell adhesion, migration and infiltration might be facilitated. He observed that other workers have shown that chronic venous reflux activates matrix metalloproteinases leading to increased extracellular matrix degradation. ${ }^{94}$

Subsequently, $109 \mathrm{MS}$ patients and 177 age-matched controls underwent transcranial and extracranial colour-Doppler sonograms. The following five findings were documented: reflux in IJV and /or vertebral veins regardless of supine or sitting position, MS patients $70 \%$, controls $0 \%$; reflux propagated to deep cerebral veins, MS patients 50\%, controls 0\%; high resolution B-mode evidence of proximal IJV stenosis, MS patients $28 \%$, controls $0.6 \%$; no detectable flow in IJV and/or vertebral veins despite numerous deep inspirations, MS patients $32 \%$, controls $0.6 \%$; negative change in cross-sectional area of IJV, MS patients $58 \%$, controls $12 \%$. In a blinded assessment a total of 1430 sonographic parameters were measured in controls, there were 861 parameters of normal venous return versus 24 anomalous. In MS cases there were 288 normal parameters and 257 abnormal. When two criteria were present 109 of 109 cases had MS and none of 177 without them had MS. The results suggested to Zamboni, 2009, that venous flow abnormalities relate only to MS and not to other neurodegenerative, immune and neurovascular diseases in which iron deposition is an epiphenomenon. ${ }^{95}$

In 2009, further evidence for his hypothesis was published by Zamboni's group. A series of 65 patients with clinical MS were compared to 235 older, healthy people, patients with other neurological diseases and patients without neurological disease who were scheduled for venography were studied. Venous outflow was studied by transcranial and extracranial colourDoppler high resolution equipment. If two of five parameters of abnormal venous flow were present they went on to venography of the IJV and AZY venous systems during which intravenous pressure measurements were taken. Patients with evidence of relapses, disease progression or steroid treatment in the previous month were excluded. Patients underwent selective venography of the lumbar, left renal, AZY and IJV. Clinical MS was associated with these cerebral venous outflow abnormalities (OR 43, 95\% CI $29-65, \mathrm{P}<0.0001)$ - an astounding correlation! Only in the MS patients did venography demonstrate the presence of severe and multiple extracranial venous stenoses. Reflux was consistently present in the IJV and/or vertebral veins in $71 \%$ of the MS cases and none of the controls. Similarly reflux in the deep cerebral veins occurred in $61 \%$ of MS cases, none of controls. There was high resolution B mode evidence of proximal IJV stenosis in $37 \%$ of MS cases and $0 \%$ of controls. The Doppler could not detect flow in the IJVs or vertebral veins (despite numerous deep inspirations at both $0^{\circ}$ and $90^{\circ}$ ) in $52 \%$ of MS patients but only 3\% of controls. Normally the crosssectional area of the IJV in the sitting position is less than in the supine so subtracting the former from the latter yields a positive value. In MS cases the majority (55\%) had a negative value versus $11 \%$ of the control population. In the MS group there was an abnormality of the AZY in $86 \%$ of cases, such as membranous obstruction at the junction with the superior vena cava, twisting, a septum or atresia. The AZY could be stenosed at several points. The lumbar plexus that drains into it was atretic in $18 \%$. The IJV were stenosed uni- or bilaterally in $91 \%$ of MS cases. The stenoses consisted of annulus and septal strictures, less frequently atresia but no twisting. In MS cases pressure in the stenotic AZY was $3.9 \mathrm{~cm} / \mathrm{H}_{2} \mathrm{O}$ higher than the adjacent superior vena cava, in the stenotic IJV it was $1.8 \mathrm{~cm} / \mathrm{H}_{2} 0$ higher. Multiple sclerosis patients showed abnormal patterns of venous return as follows: (A) (30\%) a stenosis at one IJV and the proximal AZY resulted in constant reflux in these veins, under all postural conditions, with compensatory increased flow through the unobstructed IJV and increased vertebral venous flow to the left hemiAZY vein thence to the inferior vena cava or retrograde flow cranially through the vertebral veins, in this pattern reflux was detected by Doppler in the deep middle cerebral veins in $60 \%$ of cases. (B) (38\%) significant stenosis are present in both IJV and proximal AZY, cerebral venous outflow for overcoming the stenosis is via cerebral collateral veins, for the AZY flow the return is by retrograde flow in the vertebral veins and via the hemi-AZY venous route. Eighty-three percent of relapsingremitting type of MS was associated with venous patterns of types (A) or (B). (C) (14\%) bilateral stenoses of both IJV with a normal AZY system led to reflux in the IJV and increased cervical and intracranial collateral flow into the superior vena 
cava or cranial end of the vertebral veins, thence into the AZY which is overloaded. This pattern was proportionately higher in patients with secondary progressive MS. (D) (18\%) the AZY system was affected by multiple stenoses at various segments producing retrograde flow into the vertebral veins and reversing caudal to rostral flow in them with collateral flow into the intracranial veins. This resulted in $90 \%$ visualization by Doppler of reflux flow in the deep cerebral veins. This pattern was significantly associated with spinal cord plaques and a primary progressive clinical course. The number of venous stenoses did not appear to be affected by previous therapy with immunosuppressant agents. ${ }^{96}$

The first results of therapeutic interventions based on Zamboni's hypothesis of chronic venous insufficiency were published in 2009. Sixty-five consecutive patients (35 relapsing remitting, 20 with secondary progressive and 10 primary progressive) underwent percutaneous angioplasty of strictures in the IJV and AZY. Patients with evidence of relapse, disease progression or steroid treatment in the previous month were excluded. Patients underwent selective venography of the lumbar, left renal, AZY and IJV by catheterization of the iliac femoral axis, not apparently of the superior hemiAZY or vertebral veins. These were hand injections done presumably in the supine position. A significant stenosis was defined as any vessel with a lumen reduction of $>50 \%$. Stenoses resulted from: circumferential stenosis of the entire vessel wall, septum/valve malformations, hypoplasia of long venous segments, twisting of a venous segment, membranous obstruction or agenesis of a segment. Venous stenoses of IJV were observed at the junction with the subclavian vein in $80 \%$ of right IJV, $94 \%$ of left IJV, $71 \%$ of AZY at point of entry to the superior vena cava, $23 \%$ in the course of the AZY and $9 \%$ of lumbar veins. At a mean follow-up of 18 months following the angioplasty the patency rate for IJV were $53 \%$ and for the AZY $96 \%$. The restenosis rate for the IJV (47\% at 18 months) was 16 fold higher for the IJV versus the AZY. Relapsing-remitting and secondary progressive patterns were most commonly associated with stenosis of the IJV and AZY as they joined the superior vena cava. Relapsingremitting patients had one of the stenotic patterns involving at least one IJV in 34/35, the secondary-progressive cases showed this in $19 / 20$ cases. A particular clinical pattern, primary progressive, was associated with a particular venous occlusive pattern, the multilevel stenoses of AZY and lumbar veins that was present in 9/10 cases. Post-operative supine pressure values were significantly lower in all segments, from approximately 14 to $15 \mathrm{~cm} / \mathrm{H}_{2} \mathrm{O}$ to $11 \mathrm{~cm} / \mathrm{H}_{2} \mathrm{O}$. Clinical outcome measures were consistently improved particularly in the relapsing remitting cases. The rate of relapse-free patients changed from 27 to $50 \%$ post-operatively, the first year post-percutaneous angioplasty rate was compared to the annualized average rate of relapse in the preceding two years. The MRI using gadolinium showed the number of patients with lesions went from $50 \%$ to $12 \%$ after treatment. The MS functional composite at one year was significantly better in the remitting-relapsing but not the primary progressive or secondary progressive cases. Physical and mental quality of life improved significantly in the relapsing-remitting and primary progressive cases. The procedure was safe. The high failure rate in the IJV is leading (i) to the development of shunts specifically designed to avoid pulmonary migration, (ii) the use of higher pressure angioplasty balloons or (iii) possibly open surgery. The investigators have attached the cumbersome and non-specific term "chronic craniospinal venous insufficiency" to these venous outflow abnormalities because an analogous term is used for varicose veins. Since this was an open study further prospective, randomized trials of pharmacotherapy versus percutaneous angioplasty and pharmacotherapy were called for. ${ }^{97}$

\section{Conclusion}

The possibility that venous reflux, reversal of flow, and venous hypertension are the primary inciting causes of at least some forms of MS is currently a defensible hypothesis. In varying forms this is not a new idea having some rationale from pathological studies. It remains to be seen if independent investigators, using larger numbers of patients, will replicate the strength of the association between MS and initially, sonographic abnormalities and if positive, subsequently, pathology on venography. Assuming this occurs and is also supportive, finally, interventional procedures in selected patients, enrolled in organized trials with randomization, appropriate controls, blinded assessment techniques, with indicated monitoring and sufficient follow-up time, would be appropriate. Will the operative relief of sites of flow obstruction provide durable remission? Will the procedures prove safe? Until what point in the course of the disease is it reasonable to anticipate a cessation of progression even if the theory is true? The huge community of sufferers, perhaps millions world-wide, for better or worse, already has had its hopes raised by a blizzard of one-sided reporting in the popular press and the internet on the "Liberation Procedure" so there is some urgency.

\section{REFERENCES}

1. Zamboni P. The big idea: iron-dependent inflammation in venous disease and proposed parallels in multiple sclerosis. JR Soc Med. 2006;99(11):589-93

2. Giovannoni G, Cutter GR. Infectious causes of multiple sclerosis. Lancet Neurol. 2006;5(10):887-94.

3. Ruiz DSM. Gailloud P, Rufenacht DA. The craniocervical venous system in relation to cerebral venous drainage. AJNR Am J Neuroradiol. 2002;23(9):1500-8.

4. Groen RJM, Groenewegen HJ, van Alphen HAM, et al. Morphology of the human internal vertebral venous plexus: a cadaver study after intravenous araldite CY 221. Anat Rec Part A. $1998 ; 249(2): 285-94$.

5. Lepori D, Capasso P, Fournier D, et al. High resolution ultrasound evaluation of internal jugular venous valves. Eur Radiol. 1999;9 (6):1222-6.

6. Dresser LP, Mckinney WM. Anatomic and pathophysiologic studies of the human internal jugular valve. Am J Surg. 1987; 154(2):220-4.

7. Paleri V, Gopalakrishnan S. Jugular phlebectasia: theory of pathogenesis and review of the literature. Int $\mathrm{J}$ Pediatr Otorhinolaryngol. 2001;57(2):155-9.

8. Van der Kuip M, Hoogland PV, Groen RJ. Human radicular veins: regulation of venous reflux in the absence of valves. Anat Rec. 1999;254(2):173-80.

9. Scapinelli R. Antireflux mechanisms in veins draining the upper territory of the vertebral column and spinal cord in man. Clin Anat. 2000;13(6):410-5.

10. Zamboni P, Consorti G, Galeotti R, et al. Venous collateral circulation of the extracranial cerebrospinal outflow routes. Curr Neurovasc Res. 2009;6(3):204-12. 
11. Herlihy WF. Revision of the venous system: the role of the vertebral veins. Med J Aust. 1947;1(22):661-72.

12. Pang CCY. Autonomic control of the venous system in health and disease: effects of drugs. Pharmacol Ther. 2001;90(2-3): 179-230.

13. Lin W, Celik RP, Paczynski RP, et al. Quantitative magnetic resonance imaging in experimental hypercapnia: improvement in the relation between changes in brain $\mathrm{R} 2$ and the oxygen saturation of venous blood after correction for regional blood volume. J Cereb Blood Flow Metab. 1999;19(8):853-62.

14. Schaller B. Physiology of cerebrovenous blood flow: from experimental data in animals to normal function in humans. Brain Res Rev. 2004;46(3):243-60.

15. Inao $\mathrm{S}$, Kuchiwaki $\mathrm{H}$, Yoshida J, et al. Magnetic resonance imaging quantification of superior sagittal sinus flow. Correlation to cerebral blood flow measured by Xenon enhanced computerized tomography. Neurol Res. 1997;19(1):35-40.

16. Valdueza JM, Schmierer K, Mehraein S, Einhaupl KM. Assessment of normal flow velocity in basal cerebral veins. A transcranial Doppler ultrasound study. Stroke. 1996;27(7):1221-5.

17. Gisolf J, van Lieshout J, van Keudsen K, Pott F, Stok WJ, Karemaker JM. Human jugular venous outflow pathways depend on posture and cerebral venous pressure. J Physiol. 2004 (Oct. 17);560(Pt. 1):317-27.

18. Menegatti E, Zamboni P. Doppler haemodynamics of cerebral venous return. Curr Neurovasc Res. 2008;5(4):260-5.

19. Stoquart-ElSankari S, Lehmann P, Vilette A, et al. A phase-contrast MRI study of physiological cerebral venous flow. J Cereb Blood Flow Metab. 2009;29(6):1208-15.

20. Mehta NR, Jones L, Kraut MA, et al. Physiological variations in dural venous flow on phase-contrast MR imaging. AJR Amer J Roentgenol. 2000;175(1):221-5.

21. Zervides C, Narracott AJ, Lawford PV, et al. The role of venous valves in pressure shielding. Biomed Eng OnLine. 2008;7(Feb. 15):8.

22. Buxton GA, Clarke N. Computational phlebology: the simulation of a vein valve. J Biol Physics. 2007;32(6):507-21.

23. Putnam TJ. Lesions of "encephalomyelitis" and multiple sclerosis. JAMA. 1937:108(18):1477-80.

24. Schlesinger B. The venous drainage of the brain, with special reference to the galenic system. Brain. 1939;62:274.

25. Fog T. The topography of plaques in multiple sclerosis. With special reference to cerebral plaques. Acta Neurol Scand. 1965;41 Suppl 15:1-161.

26. Scheinker M. Histogenesis of the early lesions of multiple sclerosis. 1. Significance of the vascular changes. Arch Neurol Psychiat. 1943;49:178.

27. Macchi G. The pathology of the blood vessels in multiple sclerosis. J Neuropath Exp Neurol. 1954;13(2):378-84.

28. Adams CWM, Abdulla YH, Torres EM, Poston RN. Periventricular lesions in multiple sclerosis: their perivenous origin and relationship to granular ependymitis. Neuropath Appl Neurobiol. 1987;13(2):141-52.

29. Adams CW. Vascular aspects of multiple sclerosis. Ch.8. In: Adams CW. A colour atlas of multiple sclerosis and other myelin disorders. London: Wolfe Medical Publications; 1989.

30. Barnett MH, Prineas JW. Relapsing and remitting multiple sclerosis: pathology of the newly forming lesion. Ann Neurol. 2004;55(4):458-68.

31. Lucchinetti C, Bruck W, Parisi J, Scheithauer B, Rodriguez M, Lassmann H. Heterogeneity of multiple sclerosis lesions: implications for the pathogenesis of demyelination. Ann Neurol. 2000;47(6):707-17.

32. Aboul-Enein F, Rauschka H, Kornek B, et al. Preferential loss of myelin-associated glycoprotein reflects hypoxia-like white matter damage in stroke and inflammatory brain diseases. J Neuropath Exp Neurol. 2003;62(1):25-33.

33. Aboul-Enein F, Lassmann H. Mitochondrial damage and histotoxic hypoxia: a pathway of tissue injury in inflammatory brain disease? Acta Neuropath. 2005;109(1):49-55.

34. Broman T. Blood-brain barrier damage in multiple sclerosis. Supravital test observation. Acta Neurol Scand. 1964;Suppl 10: $21-4$
35. Van Horssen J, Brink B, de Vries HE, van der Valk P, Bo L. The blood-brain barrier in cortical multiple sclerosis lesions. J Neuropath Exp Neurol. 2007;66(4):321-8.

36. Minagar A, Alexander JS. Blood-brain barrier disruption in multiple sclerosis. Mult Scler. 2003;9(6):540-9.

37. Leech S, Kirk J, Plumb J, et al. Persistent endothelial abnormalities and blood-brain barrier leak in primary and secondary progressive multiple sclerosis. J Neuropath Applied Neurobiol. 2007;33(1):86-98.

38. Van Horssen J, Vos CMP, Admiraal L, et al. Matrix metalloproteinase-19 is highly expressed in active multiple sclerosis lesions. Neuropathol App Neurobiol. 2006;32(6): 585-93.

39. Comabella M, Rio J, Espejo $\mathrm{C}$, et al. Changes in matrix metalloproteinases and their inhibitors during interferon-beta treatment in multiple sclerosis. Clin Immunol. 2009;130(2): $145-50$.

40. Haacke EM, Cheng NYC, House MJ, et al. Imaging iron stores in the brain using magnetic resonance imaging. Mag Res Imag. 2005(1);23:1-25

41. Craelius W, Migdal MW, Luessenhop CP, Sugar A, Mihalakis I. Iron deposits surrounding multiple sclerosis plaques. Arch Pathol Lab Med. 1982;106(8):397-9.

42. LeVine SM. Iron deposits in multiple sclerosis and Alzheimer's disease brains. Brain Res. 1997; 760(1-2):298-303.

43. Van Horssen J, Schreibelt G, Drexhage J, et al. Severe oxidative damage in multiple sclerosis lesions coincides with antioxidant enzyme expression. Free Radic Biol Med. 2008; 45(12):1729-37.

44. Pender MP, Greer JM. Immunology of multiple sclerosis. Curr Allergy Asthma Rep. 2007; 7(4):285-92.

45. Grant SM, Wiesiger JA, Beard JL, Cantorna MT. Iron-deficient mice fail to develop autoimmune encephalomyelitis. J Nutr. 2003;133(8):2635-8

46. Mitchell KM, Dotson AL, Cool KM, Chakrabarty A, Benedict SH, LeVine SM. Deferiprone, an orally deliverable iron chelator, ameliorates experimental autoimmune encephalomyelitis. Mult Scler. 2007;13(9):1118-26.

47. Varga AW, Johnson G, Babb JS, Herbert J, Grossman RI, Inglese M. White matter hemodynamic abnormalities precede subcortical gray matter changes in multiple sclerosis. J Neurol Sci. 2009; 282(1-2):28-33.

48. Rashid W, Parkes LM, Ingle GT, et al. Abnormalities of cerebral perfusion in multiple sclerosis. J Neurol Neurosurg Psychiat. 2004;75(4): 1288-93

49. Law M, Saindane AM, Ge Y, et al. Microvascular abnormality in relapsing-remitting multiple sclerosis: perfusion MR imaging findings in normal-appearing white matter. Radiology. 2004; 231(3):645-52.

50. Wuerfel J, Bellmann-Strobl J, Brunecker P, et al. Changes in cerebral perfusion precede plaque formation in multiple sclerosis: a longitudinal perfusion MRI study. Brain. 2004;127 (Pt 1):111-19.

51. Drayer B, Burger P, Hurwitz B, Dawson S, Cain J. Reduced signal intensity on MR images of thalamus and putamen in multiple sclerosis: increased iron content? AJR Am J Roentgenol. 1987; 149(2):357-363

52. Neema M, Stankiewicz J, Arora A, et al. T1- and T2- based MRI measures of diffuse gray matter and white matter damage in patients with multiple sclerosis. J Neuroimag. 2007;17 Suppl 1: $16 \mathrm{~S}-21 \mathrm{~S}$

53. Ge Y, Jensen JH, Lu H, et al. Quantitative assessment of iron accumulation in deep gray matter of multiple sclerosis by magnetic field correlation imaging. AJNR Amer J Neuroradiol. 2007;28(9): 1639-44

54. Haacke EM, Makki M, Ge Y, et al. Characterizing iron deposition in multiple sclerosis lesions using susceptibility weighted imaging. J Mag Res Imag 2009;29(3):537-44.

55. Ceccarelli A, Filippi M, Neema M, et al. T2 hypointensity in the deep grey matter of patients with benign multiple sclerosis. Mult Scler. 2009;15(6):678-86 
56. Khalil M, Enzinger C, Langkammer C, et al. Quantitative assessment of brain iron by $\mathrm{R} 2 *$ relaxometry in patients with clinically isolated syndrome and relapsing-remitting multiple sclerosis. Mult Scler. 2009;15(9):1048-54.

57. Hammond KE, Metcalf M, Carvajal, et al. Quantitative in vivo magnetic resonance imaging of multiple sclerosis at 7 Tesla with sensitivity to iron. Ann Neurol 2008;64(6):797-13.

58. Zivadinov R, Schirda C, Dwyer MG, et al. Chronic cerebrospinal venous insufficiency and iron deposition on susceptibilityweighted imaging in patients with multiple sclerosis: a pilot case-control study. Int Angiol. 2010;29(2):158-75.

59. Tan IL, van Schijndel RA, Pouwels MA, et al. MR venography of multiple sclerosis. AJNR Am J Neuroradiol. 2000:21(6): 1039-42.

60. Tallantyre EC, Brooks MJ, Dixon JE, et al. Demonstrating the perivascular distribution of MS lesions in vivo with 7-Tesla MRI. Neurology. 2008;70(22):2076-8

61. Hojnacki D, Zamboni P, Lopez-Soriano A, et al. Use of neck magnetic resonance venography, soppler sonography and selective venography for diagnosis of chronic cerebrospinal venous insufficiency: a pilot study in multiple sclerosis patients and healthy controls. Int Angiol. 2010;29(2):127-39.

62. Tartaglino LM, Friedman DP, Flanders AE, et al. Multiple sclerosis in the spinal cord: MR appearance and correlation with clinical parameters. Radiology. 1995;195(3):725-32.

63. Aaslid R, Newell DW, Stooss R, Sorteberg W, Lindegaard KF. Assessment of cerebral autoregulation dynamics from simultaneous arterial dynamics from simultaneous arterial and venous intracranial Doppler recordings in humans. Stroke. 1991;22(9):1148-54.

64. Stolz E, Kaps M, Korn A, Babaccan SS, Dorndorf W. Transcranial color-coded duplex sonography of intracranial veins and sinuses of adults. Reference data from 130 volunteers. Stroke. 1999;30 (5):1070-5.

65. Baumgartner RW, Gonner F, Arnold M, Muri RM. Transtemporal power- and frequency-based color-coded duplex sonography of cerebral veins and sinuses. AJNR Am J Neuroradiol. 1997;18 (9):1771-81.

66. Doepp F, Schreiber SJ, von Munster T, et al. How does blood leave the brain? A systematic ultrasound analysis of cerebral venous drainage patterns. Neuroradiol. 2004;46(7):565-70.

67. Silva MA, Deen KI, Fernando DJS, Sheriffdeen AH. The internal jugular veins valve may have a significant role in the prevention of venous reflux: evidence from live and cadaveric human subjects. Clin Physiol Funct Imag. 2002;22:202-5.

68. Brownlow RL Jr, McKinney WM. Ultrasonic evaluation of jugular venous valve competence. J Ultrasound Med. 1985; 4(4):169-72.

69. Akkawi NM, Agosti C, Borroni B, et al. Jugular valve incompetence: a study using air contrast ultrasonography on a general population. J Ultrasound Med. 2002;21(7):747-51.

70. Chung C-P, $\mathrm{Hu} \mathrm{H}-\mathrm{H}$. Jugular venous reflux. J Med Ultrasound. 2008;16(3):210-22.

71. Chou $\mathrm{CH}$, Chao AC, Hu HH. Ulrasonographic evaluation of vertebral venous valves. AJNR Am J Neuroradiol. 2002;23(8): $1418-20$.

72. Nedelmann M, Eicke BM, Dieterich M. Functional and morphological criteria of internal jugular valve insufficiency as assessed by ultrasound. J Neuroimaging. 2005;15(1):70-5 .

73. Doepp F, Schreiber SJ. Benndorf G, Radtke A, Gallinat J, Valdueza $\mathrm{JM}$. Venous drainage patterns in a case of pseudotumor cerebri following unilateral radical neck dissection. Acta Otolaryngol. 2003;123(8):994-7.

74. Humphries WE, Grossi PM, Liethe LG, et al. Ventriculoperitoneal shunt failure causing myelopathy in a patient with bilateral jugular vein occlusions. Case Report. J Neurosurg Spine. 2007;6 (1):60-3.

75. Huang P, Yang Y, Chen R, et al. Successful treatment of cerebral venous thrombosis associated with bilateral internal jugular vein stenosis using direct thrombolysis and stenting: a case report. Kaohsiung J Med Sci. 2005;21(11):527-31.
76. Barrett N, Spencer S, McIvor J, et al. Subclavian stenosis: a major complication of subclavian dialysis catheters. Nephrol Dial Transplant. 1998;3(4):423-5.

77. Schillinger F, Schillinger D, Montagnac R, et al. Post catheterization vein stenosis in haemodialysis: comparative angiographic study of 50 subclavian and 50 internal jugular accesses. Nephrol Dial Transplant. 1991;6(10):722-4.

78. Taal MW, Chesterton LJ, McIntyre W. Venography at insertion of tunneled internal jugular vein catheters reveals significant occult stenosis. Nephrol Dial Transplant. 2004;19(6):1542-5.

79. MacRae JM, Ahmed A, Johnson N, et al. Central vein stenosis: a common problem in patients on hemodialysis. ASAIO J. 2005;51(1):77-81.

80. Hartmann A, Mast M, Stapf C, Kock H-C, Marx P. Peripheral hemodialysis shunt with intracranial venous congestion. Case Reports. Stroke. 2001;32(12):2945-6.

81. Orin PR. Sonology demonstrates flow reversal within internal jugular vein secondary to occluded innominate vein and stenotic hemodialysis arteriovenous fistula. J Diag Med Sonol. 2002;18 (6):405-8

82. Paksoy Y, Genc BO, Genc E. Retrograde flow in the left inferior petrosal sinus and blood steal of the cavernous sinus associated with central vein stenosis: MR angiographic findings. AJNR Am J Neuroradiol. 2003;24(7):1364-8.

83. Doepp F, Bahr D, John M, Hoernig S, Valdueza JM, Schreiber SJ. Internal jugular vein valve incompetence in COPD and primary pulmonary hypertension. J Clin Ultrasound. 2008;36(8):480-4.

84. Doepp F, Valdueza JM, Schreiber SJ. Incompetence of internal jugular valve in patients with primary exertional headache: a risk factor? Cephalalgia. 2008;28:182-5.

85. Nedelmann M, Eicke BM, Dieterich M. Increased incidence of jugular valve insufficiency in patients with transient global amnesia. J Neurol. 2005;252(12):1482-6.

86. Altamura $\mathrm{C}$, Vernieri $\mathrm{F}$. Internal jugular vein incompetence in transient global amnesia. More circumstantial evidence or the proof solving the mystery? Stroke. 2010;41(1): 1

87. Taylor GA, Walker LK. Intracranial venous system in newborns treated with extracorporeal membrane oxygenation: doppler ultrasound evaluation after ligation of right internal jugular vein. Radiology. 1999;183(2):453-6.

88. Wilkinson N, Sood S, Ham SD, et al. Thrombosis associated with ventriculoatrial shunts. J Neurosurg Pediatr. 2008;2(4):286-91.

89. Leriche H, Aubin ML, Abouker J. [ Cavo-spinal phlebography in myelopathies. Stenoses of internal jugular and azygous veins, venous compressions and thromboses.] [Article in French] Acta Radiol Suppl. 1976;347:415-7.

90. Anonymous. Retinal veins in multiple sclerosis. BMJ. 1972(16 Dec):626.

91. Rosales Carbella J. [The significance of enlarged retinal veins in the diagnosis of multiple sclerosis: report of a patient] [Article in Spanish] Med Clin (Barc). 1981;77(1):30-2.

92. Lightman S, McDonald WI, Bird AC, et al. Retinal venous sheathing in optic neuritis. Its significance for the pathogenesis of multiple sclerosis. Brain. 1987;110 Pt 2:405-14.

93. Bergan JJ, Schmid-Schonbein GW, Coleridge Smith PD, Nicolaides AN, Boisseau MR, Eklof B. Chronic venous disease. N Engl J Med. 2006;355(5):488-98.

94. Zamboni P, Menegatti E, Bartolomei I, et al. Intracranial venous haemodynamics in multiple sclerosis. Curr Neurovasc Res. 2007;4(4):252-8

95. Zamboni P, Menegatti E, Galeotti R, et al. The value of cerebral Doppler venous haemodynamics in the assessment of multiple sclerosis. J Neurol Sci. 2009;282(1-2):21-7.

96. Zamboni P, Galeotti R, Menegatti E, et al. Chronic cerebrospinal venous insufficiency in patients with multiple sclerosis. J Neurol Neurosurg Psychiat. 2009;80(4):392-9.

97. Zamboni P, Galeotti R, Menegatti E, et al. A prospective open-label study of endovascular treatment of chronic cerebrospinal venous insufficiency. J Vasc Surg. 2009;50(6):1348-58 